\title{
EC-QC laser spectroscopy for mid-IR transmission measurements of proteins in aqueous solution
}

\author{
Mirta R. Alcaráz, ${ }^{\dagger+}$ Andreas Schwaighofer, ${ }^{\dagger}$ Christian Kristament, ${ }^{\dagger}$ Georg Ramer, ${ }^{\dagger}$ Markus Brand- \\ stetter, ${ }^{\dagger,}$ Héctor Goicoechea, ${ }^{\dagger}$ Bernhard Lendl*, + \\ IInstitute of Chemical Technologies and Analytics, Vienna University of Technology, Getreidemarkt 9/164-UPA, 1060 \\ Vienna, Austria \\ \$Laboratorio de Desarrollo Analítico y Quimiometría (LADAQ), Cátedra de Química Analítica I, Facultad de Bioquímica \\ y Ciencias Biológicas, Universidad Nacional del Litoral-CONICET, Ciudad Universitaria, 3000 Santa Fe, Argentina
}

This document is the unedited Author's version of a Submitted Work that was subsequently accepted for publication in Analytical Cheimstry copyright (C) American Chemical Society after peer review. To access the final edited and published work see https://pubs.acs.org/doi/10.1021/acs.analchem.5b01738

\begin{abstract}
In this work we report mid-IR transmission measurements of the protein amide I band in aqueous solution at large optical paths. A tunable external-cavity quantum cascade laser (EC-QCL) operated in pulsed mode at roomtemperature allowed applying path length of up to $38 \mu \mathrm{m}$, which is four times larger than applicable with conventional FTIR spectrometers. To minimize temperature-induced variations caused by background absorption of the $v_{2}$-vibration of water (HOH-bending) overlapping with the amide I region, a highly-stable temperature control unit with relative temperature stability within $0.005^{\circ} \mathrm{C}$ was developed. An advanced data processing protocol was established to overcome fluctuations in the fine structure of the emission curve that are inherent to the employed EC-QCL due to its mechanical instabilities. To allow for wavenumber accuracy, a spectral calibration method has been elaborated to reference the acquired IR spectra to the absolute positions of the water vapor absorption bands. Employing this setup, characteristic spectral features of five wellstudied proteins exhibiting different secondary structures could be measured at concentrations as low as $2.5 \mathrm{mg}$ ml-1. This concentration range could previously only be accessed by IR measurements in $\mathrm{D}_{2} \mathrm{O}$. Mathematical evaluation of the spectral overlap and comparison of second derivative spectra confirm excellent agreement of the QCL transmission measurements with protein spectra acquired by FTIR spectroscopy. This proves the potential of the applied setup to monitor secondary structure changes of proteins in aqueous solution at extended optical path lengths, which allow experiments in flow through configuration.
\end{abstract}

Infrared spectroscopy is a versatile technique for the quantitative and qualitative analysis of a wide range of samples. After the development of the fast Fourier transform algorithm in the $1960,{ }^{1}$ it was feasible to employ the Michelson-interferometer for IR spectroscopy. Since then, Fourier transform infrared (FTIR) spectrometers have been established as standard instrumentation. Thermal emitters such as globars are commonly used as light sources that emit a broadband but low-power radiation throughout the mid-IR region. While these characteristics suffice for a variety of applications, the low emission intensity of the light source constitutes a limit for the measurement of samples with a highly absorbing matrix, e.g. water. In these cases, the abundant solvent absorbs a large part of the irradiated light, thus attenuating the light intensity which is available for interaction with the actual analyte. As a consequence the maximum feasible path length for transmission measurements in aqueous solution is limited in the carbohydrate region (950-1200 $\mathrm{cm}^{-1}$ ) to approx. $80 \mu \mathrm{m}$ and in the amide I region of proteins $\left(1600-1700 \mathrm{~cm}^{-1}\right)$ to approx. $8 \mu \mathrm{m} .{ }^{2,3}$ These low path lengths may considerably impair the robustness of the method due to clogging of the cell.

Two decades ago, a new type of mid-IR light source, quantum cascade lasers (QCLs) were introduced. ${ }^{4}$ QCLs provide spectral power densities that are several orders of magnitudes higher than globars and even surpass values of synchrotrons. ${ }^{5}$ QCLs comprise a series of layers of different semiconductor materials exhibiting varying band gaps with a defined thickness in the nanometer range. Laser emission is achieved by inter sub-band transitions of electrons within the semiconductors conduction band. Only one type of charge carrier (electrons) is used contrary to conventional semiconductor lasers, where electrons and holes from the conduction and valence band combine in a radiative inter-band transition. In conventional lasers, the emission wavelength is determined by the band gap of the utilized materials, thus restricting their application to $\mathrm{UV} / \mathrm{Vis}$ and near-IR range. In QCLs, the emission wavelength is decoupled from the band gap of the semiconductor material and lasers emitting at a desired wavelength region can be engineered by tuning the thicknesses of the semiconductor layers. ${ }^{6-8}$ Initially, QCLs were predominantly used for gas phase analysis. ${ }^{9-12}$ Distributed feedback (DFB) QCLs feature a grating on top of the active semiconductor material for selection of the desired wavenumber. Limited tunability across a few wavenumbers is adequate for gas phase analysis, where spectra are dominated by narrow, well resolved bands. This is not the case for the 
investigation of liquid samples, where absorption bands are much broader and overlapping of spectral components may occur, too. Hence, for the analysis of liquids, a broader spectral range is essential. Recently, external-cavity QCLs (EC-QCLs) became commercially available, that are operated at room-temperature and combine high spectral power densities with a large spectral tuning range. Here, an external cavity facilitates wavenumber selection by an external diffraction grating. Changing the angle of the diffraction grating relative to the QCL chip allows for tuning ranges of up to several hundred wavenumbers. This type of QCL was applied for analysis of complex mixtures of analytes in aqueous solution in online process monitoring, ${ }^{13}$ and for medical applications. ${ }^{2,14-16}$ High emission intensities made possible to employ larger path length for liquid transmission measurements and by that enhancing the ruggedness of the resulting analysis system compared to FTIR spectroscopy.

Infrared spectroscopy is a well-established experimental technique for the secondary structure analysis of proteins. ${ }^{17}$ The most prominent absorption bands of proteins in the IR spectrum are caused by vibrations of the peptide group and are referred to as amide bands. Among these characteristic bands in the mid-IR region, the amide I band (1600-1700 $\mathrm{cm}^{-1}$ ) which is mainly composed of $\mathrm{C}=0$ stretching vibrations is most commonly used for analysis of the secondary structure. ${ }^{18}$ The differing pattern of hydrogen bonding, dipole-dipole interactions and geometric orientations in the $\alpha$-helices, $\beta$-sheets, turns and random coil structures induce different frequencies of the $\mathrm{C}=\mathrm{O}$ vibrations that can be correlated with the respective secondary structural folding. ${ }^{19}$ Quantitative estimation of protein secondary structure has often been performed by curve fitting of the amide I band. This approach, however, often lacks in accuracy and its applicability is limited as it requires a series of subjective decisions ${ }^{18,20}$ and has a tendency to over- or underestimate secondary structure components. $^{21,22}$ Transmission measurements are most frequently used for recording solution spectra of proteins, while the attenuated total reflectance (ATR) method is employed for adsorption studies or investigation of thin films.

The most important difficulty of IR investigations of proteins in aqueous solution is the strong absorbance of the $\mathrm{HOH}$-bending band near $1645 \mathrm{~cm}^{-1}$ that overlaps with the amide I band. Consequently, suitable path lengths are restricted to approx. $8 \mu \mathrm{m}$ to prevent total IR absorption in the region of the $\mathrm{HOH}$-bending band, as outlined above. Thus, high protein concentrations $\left(>10 \mathrm{mg} \mathrm{mL}^{-1}\right)$ are required as the intensities of the IR bands and the signal-tonoise ratio at a given concentration is limited. ${ }^{18}$ Cell and sample handling under these conditions is rather laborious as thin spacers are extremely susceptible to electrostatic charging making the assembly of a tight flow cell difficult. Further, highly concentrated protein solutions are viscous leading to the formation of air bubbles when filling the cell. ${ }^{3}$ In this context, a further experimental challenge for obtaining appropriate protein spectra is to ensure experimental conditions that are identical for the background and sample measurement. Since changing temperatures cause variations in the $\mathrm{HOH}$-bending band, temperatures of the aqueous buffer and sample solution should match within at least $0.1{ }^{\circ} \mathrm{C}$ in order to avoid artifacts caused by temperature differences.

Alternatively, $\mathrm{D}_{2} \mathrm{O}$ is widely used as solvent for protein measurements. The DOD-bending band is located at $\sim 1200 \mathrm{~cm}^{-1}$, thus creating a region of relatively low solvent absorbance between 1500 and $1800 \mathrm{~cm}^{-1}$. This allows for larger path length $(\sim 50 \mu \mathrm{m})$ and lower protein concentrations $\left(\sim 1 \mathrm{mg} \mathrm{mL}^{-1}\right)$. Solvent exchange $\left(\mathrm{H}_{2} \mathrm{O} \rightarrow \mathrm{D}_{2} \mathrm{O}\right)$ leads to a shift of the amide I band to lower wavenumbers due to the replacement of hydrogen atoms in the protein backbone by heavier deuterium atoms. ${ }^{18,23}$ Despite the experimental advantages offered by $\mathrm{D}_{2} \mathrm{O}$, it is still preferable to use $\mathrm{H}_{2} \mathrm{O}$ when studying the protein structure, as it provides the native environment. ${ }^{3,24,25}$ The effect on H-D exchange on protein structure is not fully resolved but it has been reported that exposure of proteins to $\mathrm{D}_{2} \mathrm{O}$ may alter the strength and length of hydrogen bonds and leads to changes in structure and protein dynamics ${ }^{26}$ as well as protein denaturation. ${ }^{27,28}$

In this work we introduce a rugged setup based on an ECQCL for mid-IR transmission measurements of aqueous protein solutions in the amide I region. The high emission intensity of the tunable QCLs enabled increasing the path length up to $38 \mu \mathrm{m}$. A custom-built temperature control unit was designed that provides a relative temperature accuracy of the measurement cell in the order of $0.005^{\circ} \mathrm{C}$ to minimize temperature-induced variations of the $\mathrm{HOH}$ bending band in the spectrum. The focus of this paper is to demonstrate the feasibility of this setup to elucidate the spectral features of different protein secondary structures. For this purpose, spectra of five proteins with well-known secondary structures were recorded with a QCL-based transmission setup and contrasted with FTIR spectra. Evaluation by second-derivative spectra confirmed excellent comparability of the acquired spectra.

\section{EXPERIMENTAL SECTION}

Reagents and samples. Sodium phosphate monobasic dihydrate p.a. $\left(\mathrm{NaH}_{2} \mathrm{PO}_{4} \bullet 2 \mathrm{H}_{2} \mathrm{O}\right)$ was purchased from Fluka (Buchs, Switzerland), sodium phosphate dibasic dihydrate $\left(\mathrm{Na}_{2} \mathrm{HPO}_{4} \bullet 2 \mathrm{H}_{2} \mathrm{O}\right)$ BioUltra, for molecular biology, and sodium hydroxide solution $50 \%$ in water, were obtained from Sigma-Aldrich (Steinheim, Germany). $\beta$-Lactoglobulin from bovine milk ( $\geq 85 \%$ ), hemoglobin from bovine blood, bovine serum albumin ( $\geq 98.0 \%)$, lysozyme from chicken egg white $(>90 \%)$ and $\alpha$-chymotrypsin from bovine pancreas ( $\geq 85 \%$ ) were obtained by Sigma-Aldrich (Steinheim, Germany) and used as purchased. Appropriate amount of lyophilized protein powder was dissolved in $16.0 \mathrm{mmol} \mathrm{L}^{-1}$ phosphate buffer $\mathrm{pH}$ 7.0. Ultrapure water (18 $\mathrm{M} \Omega$ ) was used for preparation of all solutions, obtained with a MilliQ water purification system from Millipore (Bedford, USA).

FTIR measurements. FTIR absorption measurements were performed using a Bruker $80 \mathrm{v}$ FTIR spectrometer (Ettlingen, Germany) equipped with a liquid nitrogen cooled HgCdTe (Mercury cadmium Telluride) detector $\left(D^{*}=4 \times 10^{10} \mathrm{~cm} \mathrm{~Hz}^{0.5} \mathrm{~W}^{-1}\right.$ at $\left.9.2 \mu \mathrm{m}\right)$. The samples were placed between two $\mathrm{CaF}_{2}$ windows separated by an $8 \mu \mathrm{m}$ thick spacer. During measurements, the spectrometer was flushed with dry air for at least 10 minutes prior to spectrum acquisition. 


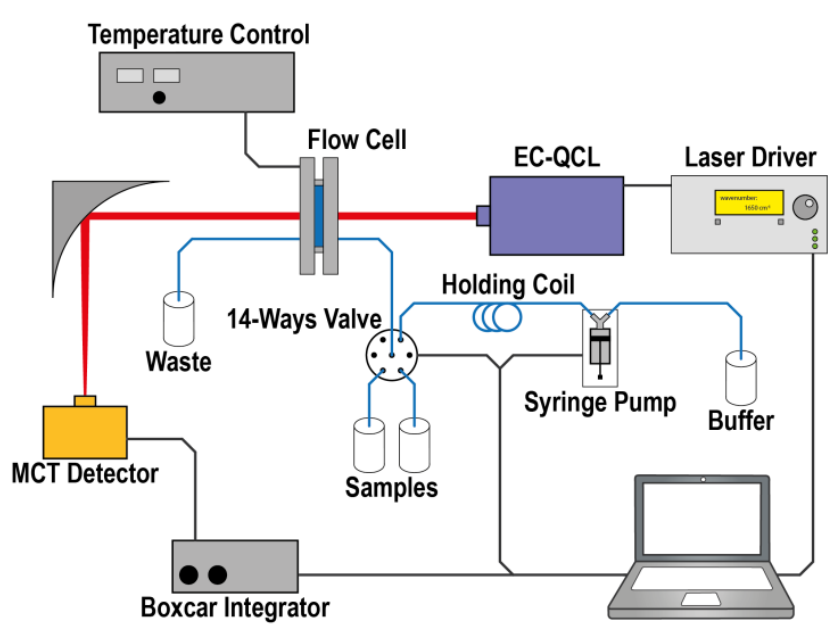

Figure 1. Representation of the experimental QCL-based setup for mid-IR transmission measurements.

Spectra were acquired with a spectral resolution of $2 \mathrm{~cm}^{-1}$ in double-sided acquisition mode. A total of 32 scans were averaged per spectrum (acquisition time: $6.7 \mathrm{~s}$ ), which was calculated using a Blackman-Harris 3-term apodization function and a zero filling factor of 2 . All spectra were acquired at $25^{\circ} \mathrm{C}$. Spectra were analyzed using the software package OPUS 7.2 (Bruker, Ettlingen, Germany). If necessary, absorption bands of water vapor in the atmosphere were subtracted.

Experimental setup for QCL measurements. The experimental setup is shown in Figure 1. For the measurements a thermoelectrically cooled external-cavity quantum cascade laser (Daylight Solutions Inc., San Diego, USA) was used operating at a repetition rate of $100 \mathrm{kHz}$ and a pulse width of $500 \mathrm{~ns}$. The manufacturer states laser power variations of $<2 \%$ RMS and an operating temperature range of $15-35^{\circ} \mathrm{C}$. The laser head temperature was set to $18{ }^{\circ} \mathrm{C}$ for all measurements. All spectra were recorded in the spectral tuning range located between 1729.30$1565.06 \mathrm{~cm}^{-1}$, covering the amide I region of proteins. The relation between the spectral tuning time and the corresponding emission wavenumber was determined through characterization of laser tuning using the step scan capability of the employed FTIR spectrometer. ${ }^{15}$ The MIR light was focused on the detector element by a gold plated offaxis parabolic mirror with a focal length of $43 \mathrm{~mm}$. A thermoelectrically cooled MCT detector operating at $-60^{\circ} \mathrm{C}$ (Infrared Associates Inc., USA; MCT-7-TE3) with a $1 \times 1 \mathrm{~mm}$ element size and a detectivity of $D^{*}=4 \times 10^{9} \mathrm{~cm} \mathrm{~Hz}^{0.5} \mathrm{~W}^{-1}$ at $9.2 \mu \mathrm{m}$ was used as IR detector.

The measured signal was processed by a two channel boxcar integrator digitized by a NI DAQ 9239 24-bit ADC (National Instruments Corp., Austin, USA). Each single beam spectrum consisting of 24000 data points was recorded during the tuning time of $1.5 \mathrm{~s}$. A total of 50 scans were recorded for background and sample single beam spectra. An AD7760 24-bit ADC (Analog Devices, Norwood, USA) was employed to investigate the relative wavenumber shifting.

The spectral resolution of the EC-QCL setup was determined by evaluation of the band width of water vapor spectra

and

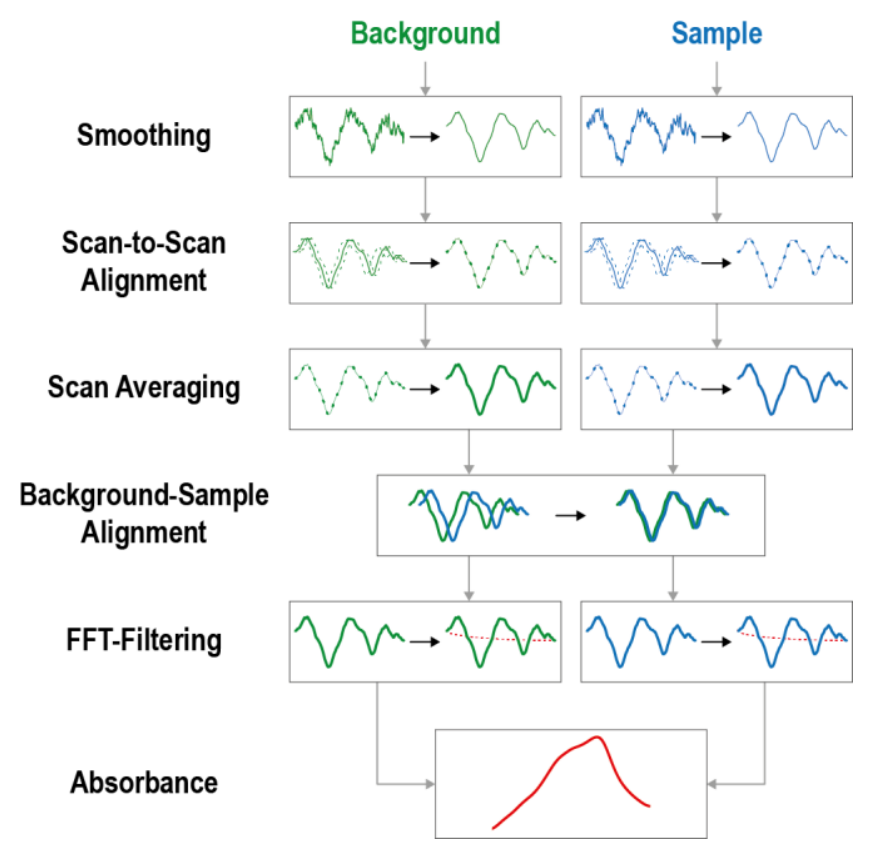

Figure 2. Sequence of processing steps for EC-QCL IR transmission spectra.

comparison with FTIR spectra acquired at different resolutions. After applying scan-to-scan alignment and background-sample alignment (see next section), the resolution of EC-QCL spectra was determined to be $0.2 \mathrm{~cm}^{-1}$ and $1.2 \mathrm{~cm}^{-1}$ for non-filtered and filtered spectra, respectively.

All measurements were carried out using a custom-made, temperature-controlled flow cell equipped with two MIR transparent $\mathrm{CaF}_{2}$ windows and $38 \mu$ m-thick spacer, at approx. $24.6{ }^{\circ} \mathrm{C}$ with relative temperature stability within $0.005^{\circ} \mathrm{C}$. The temperature cell consists of nine thermoelectric cooling (TEC) elements, stabilized by liquid water cooling and can be operated from $0-80^{\circ} \mathrm{C}$. A sensor is located near the $\mathrm{CaF}_{2}$ windows to provide constant monitoring of the temperature. The cell is equipped with a meander $(1565 \mathrm{~mm})$ to provide temperature stabilization for flow through measurements. To reduce the influence of water vapor, the setup was placed in a housing of polyethylene foil and constantly flushed with dry air. Automatic sampling was done by a flow injection system, consisting of a 14-way valve (Valco Instruments Co. Inc, Schenkon, Switzerland), a $2.5 \mathrm{~mL}$ syringe pump (Cavro XP3000 Tecan Systems Inc., Männedorf, Switzerland) and PTFE tubings in several dimensions. The whole setup was controlled by a LabView-based GUI (National Instruments Corp., Austin, USA) with server-client program structure. ${ }^{29}$ The dimensions of the setup including electronic parts are $58 \mathrm{~cm} \times 47 \mathrm{~cm} \times 77 \mathrm{~cm}$ ( L x W x H).

Processing of QCL Data. The performed preprocessing steps are schematically depicted in Figure 2. First, the number of data points in the single beam was reduced by factor 4 using cubic spline interpolation followed by Savitzky-Golay smoothing to reduce instrumental noise. To correct the spectral mismatch of successive scans (see discussion for details), Correlation Optimized Warping (COW) was applied to align consecutive scans of one measurement prior to averaging as well as to align the background with the sample single beam spectrum. Scanto-scan alignment was performed to 50 single beam spec 


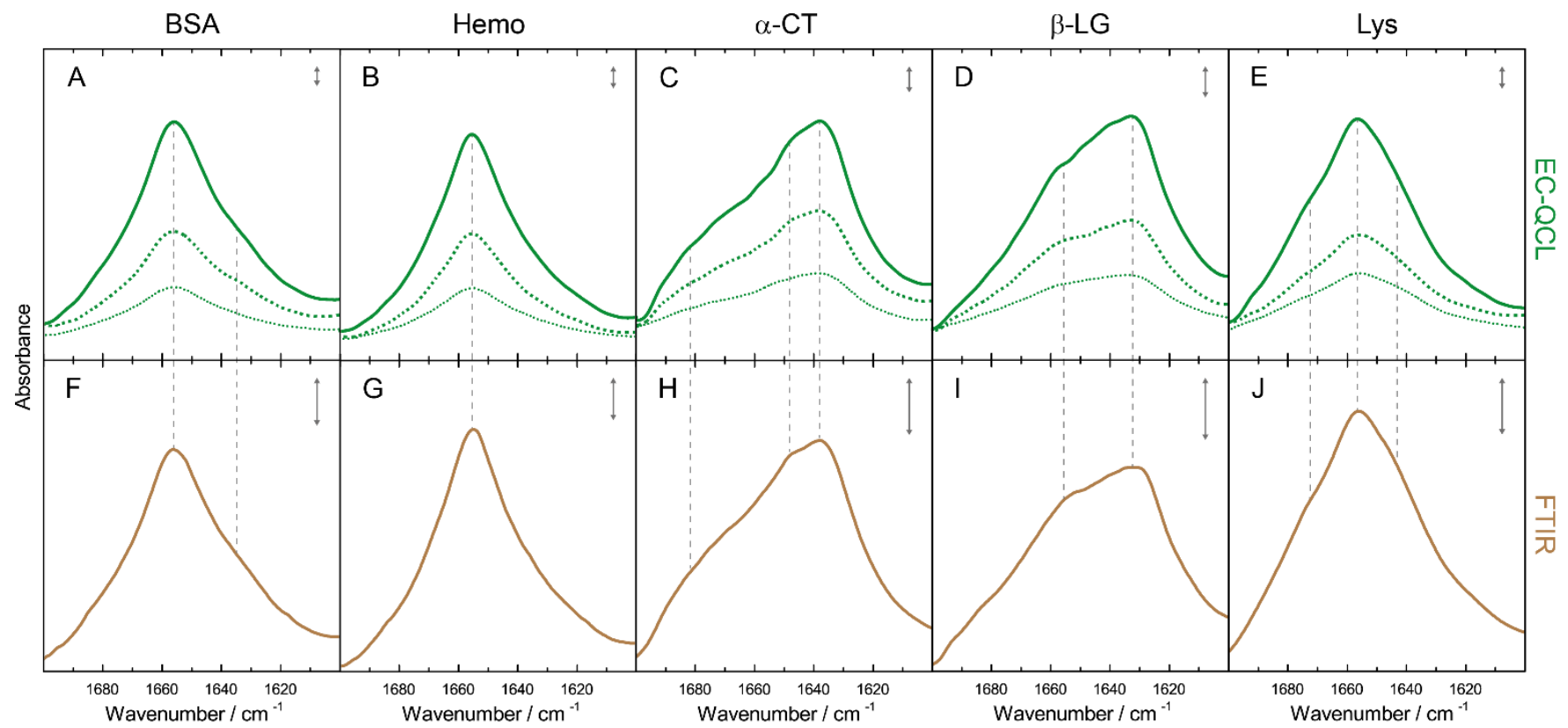

Figure 3. IR absorbance spectra of 10 (green solid line), 5 (green dashed line) and $2.5 \mathrm{mg} \mathrm{mL}^{-1}$ (green dotted line) protein solutions acquired by the EC-QCL setup (A-E) and $20 \mathrm{mg} \mathrm{m}^{-1}$ (brown solid line) protein solutions acquired by FTIR spectroscopy (F-J). Grey dashed lines highlight the high congruence of the spectral features between the IR spectra acquired by EC-QCL and FTIR spectroscopy. Grey double-headed arrows indicate the absorbance of $10 \mathrm{mAU}$.

tra, taking the middle spectrum as reference. After alignment, the spectra were averaged. Background ( $\mathrm{I}_{0}$ )sample (I) alignment was applied taking the backgroundspectrum as reference. COW is a widely used method to correct spectral (NMR, IR or Raman) or chromatographic shifts. Two parameters have to be set, the segment size $r$ and a slack size $l$. Considering two spectra, one is chosen as the reference. The second spectrum is subdivided into sections of length $r$ which are iteratively stretched and compressed for maximally $l$ data points via interpolation so as to maximize the correlation between the spectra. This kind of alignment does neither distort the amplitude nor the wavenumber position of the final absorbance spectrum..$^{30}$ In this work, $r$ was kept constant at 20 data points (corresponding $0.45 \mathrm{~cm}^{-1}$ ) and selected considering the length of mode-hops structure and $l$ was selected and optimized within the range of 2-5 data points for each sample.

After alignment of the sample and background spectrum, a low-pass Fourier filter based on 4-term Blackman-Harris apodization (Fast Fourier Transformation-FFT) was applied to single beam spectra I and $\mathrm{I}_{0}$. The final absorption spectrum was obtained using

$$
\mathrm{A}=-\log \left(\frac{I}{I_{0}}\right)
$$

In order to quantitatively evaluate the comparability of the protein IR spectra acquired by EC-QCL and FTIR spectroscopy by the degree of spectral overlap $\left(s_{12}\right)$ between FTIR $\left(\mathbf{s}_{1}\right)$ and EC-QCL $\left(\mathbf{s}_{2}\right)$, the following expression was employed: ${ }^{31}$

$$
s_{12}=\frac{\left\|\mathbf{s}_{1}^{\mathrm{T}} \mathbf{s}_{2}\right\|}{\left\|\mathbf{s}_{1}\right\|\left\|\mathbf{s}_{2}\right\|}
$$

This evaluation was performed for absorbance spectra as well as second derivative spectra. The value of $s_{12}$ ranges from 0 to 1 , corresponding to no overlapping and complete overlapping, respectively.

Data processing and analysis was performed with inhouse code developed in MatLab R2014b ${ }^{\circledR}$ (MathWorks, Inc., Natick, MA, 2014). The COW routine is available online at http://models.kvl.dk/DTW_COW.

\section{RESULTS AND DISCUSSION}

Mid-IR spectra recorded with the EC-QCL setup. Mid-IR transmission spectra were recorded of aqueous solutions of five proteins at different concentrations to assess the capabilities of the EC-QCL setup for monitoring protein secondary structure (see Figure 3A-F). The broad tuning range of the applied EC-QCL allows to record spectra across the amide I range, thus enabling quantitative as well as qualitative evaluation of the protein spectra. The investigated proteins exhibit characteristic spectral features of different secondary structures. The spectra of bovine serum albumin (BSA) and hemoglobin (Hemo), two wellstudied proteins consisting primarily of $\alpha$-helical structures, ${ }^{32,33}$ show the $\alpha$-helix characteristic band at $1656 \mathrm{~cm}^{-}$ ${ }^{1.17} \alpha$-Chymotrypsin (ACT) and $\beta$-lactoglobulin (BLG) are mainly composed of $\beta$-sheet secondary structure with a characteristic band maximum at approx. $1635 \mathrm{~cm}^{-1}$ and a side band at $1680 \mathrm{~cm}^{-1} .{ }^{32,34}$ Lysozyme (Lys) features both, $\alpha$-helices and $\beta$-sheets, resulting in a band maximum at $1656 \mathrm{~cm}^{-1}$ with shoulders at $\sim 1640 \mathrm{~cm}^{-1}$ and $\sim 1675 \mathrm{~cm}^{-}$ $1.32,35$ Spectral features of the diverse secondary structures could be identified at a concentration as low as $2.5 \mathrm{mg} \mathrm{ml}^{-1}$. This could be achieved by employing a high intensity ECQCL enabling the increased optical length of $38 \mu \mathrm{m}$ conjoined with a sophisticated data processing routine. 


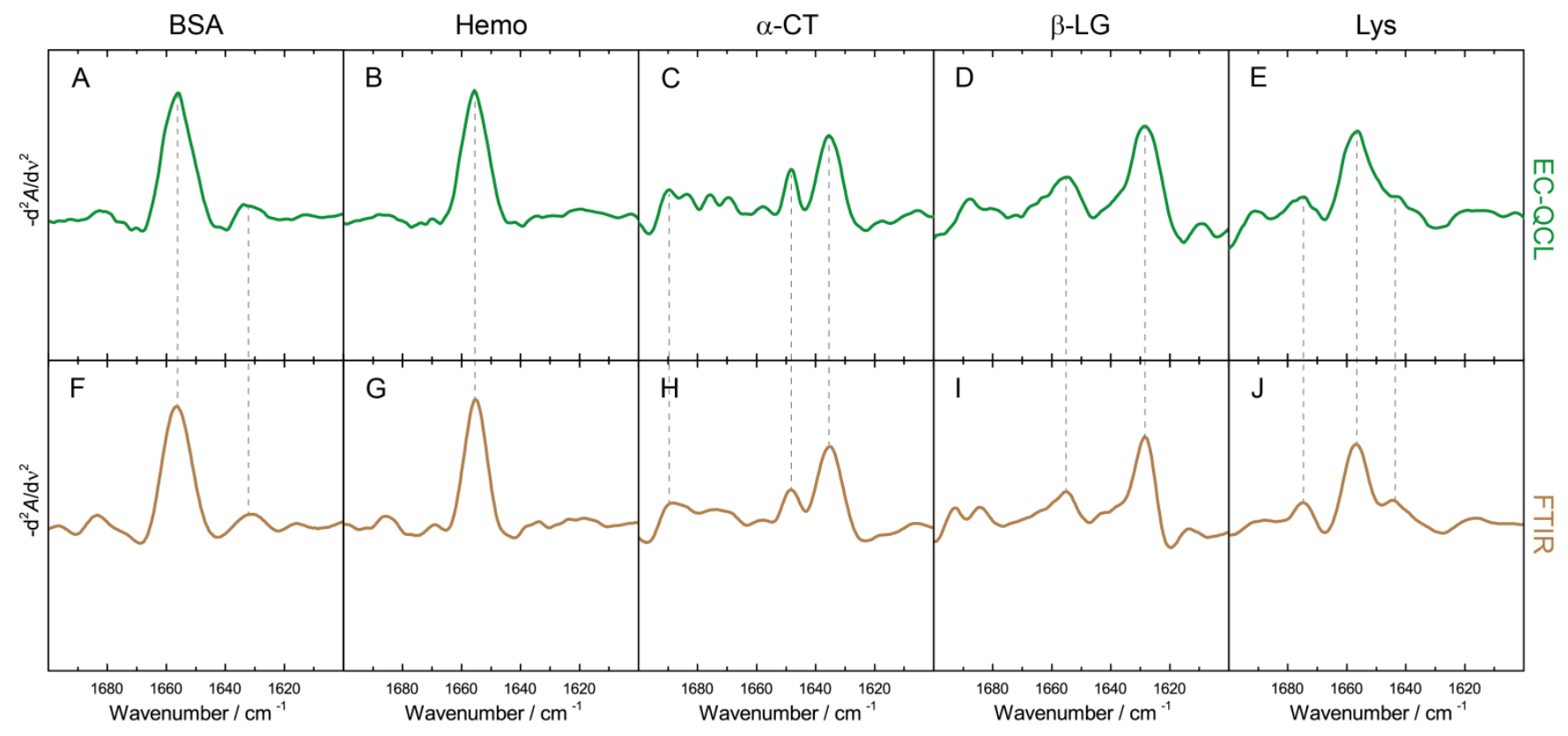

Figure 4. Inverted second derivative spectra of sample proteins acquired by the EC-QCL setup (A-E) and FTIR spectroscopy (F-J). Grey dashed lines highlight the high overlap of the spectral features between the IR spectra acquired by EC-QCL and FTIR spectroscopy.

Band areas were evaluated for proteins solutions with five concentrations of every sample. The calibration curves in Figure S-1 show straight lines and demonstrate linearity down to a concentration of $1 \mathrm{mg} \mathrm{mL}^{-1}$. The validity of the Lambert Beer law is significant for prospective applications of this setup for quantitative analysis.

Processing of raw data. In EC-QCLs, spectral tuning across the wavenumber range is achieved by a quasicontinuous rotation of the EC grating. The resulting emission curve features a fine structure originating from the mode-hops of the laser chip and external cavity. ${ }^{36}$ Mechanical imperfections as well as triggering issues lead to a spectral mismatch in the fine structure of consecutive scans (as shown in Figure S-2A). The fluctuations appear small in the single beam spectra but cause considerable noise in the corresponding absorbance spectrum. ${ }^{37}$ Since these fluctuations are not random, averaging of a high number of scans is not an option to decrease its disturbance. It had been attempted to reduce the effect of the fluctuations on the absorbance spectrum by shifting the recorded scans along the wavenumber axis to achieve maximum alignment. ${ }^{37}$ However, since the shift is not constant during one scan, even after applying this procedure, extensive use of Fourier Filtering was necessary to obtain optimal spectrum quality. Fourier filtering tends to introduce distortions in the line shape, thus affecting the information contained in the amide I band. ${ }^{38}$ To keep usage of Fourier Filtering to a minimum, an advanced spectral alignment routine was elaborated (as depicted in Figure 2). The key element of this sequence is the COW algorithm applied here to align scans prior to averaging as well as to align the background with the sample spectrum. This method utilizes the EC-QCL inherent mode-hop fine structure for alignment. It not only shifts the spectra in the wavenumber axis but also performs a rubberband type alignment. This part turned out to be crucial since the mismatch of the fine structure is not constant throughout the spectrum. A comparison between the shift- and COWbased alignment is shown in Figure S-2B. Noise reduction by shift alignment was insufficient for protein spectra. With this method, spectral features of the respective secondary structures could be recognised for protein concentrations of $10 \mathrm{mg} \mathrm{mL}^{-1}$. By introducing the COW procedure, the identification of characteristic spectral features of diverse secondary structures could be accomplished for concentrations as low as $2.5 \mathrm{mg} \mathrm{mL}^{-1}$. This improvement is mainly credited to the significantly improved reduction of noise. In consequence merely minor Fourier filtering is required, with a cutoff frequency of $50 \mathrm{~Hz}$.

Wavenumber calibration of the EC-QCL setup. The accuracy of the wavenumber scale in IR spectra has been the topic of discussion for spectroscopic instruments, ${ }^{39}$ and in particular for QCL setups. ${ }^{37}$ Deviations in the wavenumber scale may occur from inaccuracies in the light source, data acquisition as well as data processing. In EC-QCLs, one sweep of the grating in a continuous motion results in the wavenumber scan. The calibration of the wavenumber axis with tuning time for the employed EC-QCL is performed through characterization with a time-resolved (step-scan) FTIR measurement. ${ }^{15}$ Regarding data acquisition, it was found that the type of ADC has a considerable effect on the resulting IR spectrum, due to different time lags owing to triggering inaccuracies and internal data processing routines. As outlined above, the temporal axis directly corresponds to the wavenumber axis, thus time lags introduced in data acquisition translate into wavenumber deviations.

In order to correct the deviations in the wavenumber scale, the EC-QCL setup has been calibrated to the absorption lines of water vapor. Previously, a method for frequency calibration of EC-QCLs has been proposed that utilizes the inherent fine-structure superimposed to the laser emission curve. ${ }^{37}$ Here, high resolution FTIR spectra of the EC-QC 
laser emission are recorded at fixed grating positions, thus the FTIR spectrometer serves as absolute wavelength reference. A notable advantage of the calibration method employing the absorption bands of the ubiquitous water vapor as a reference is the independence from external equipment. Frequency calibration to gas-phase absorption bands has been widely applied for different kinds of spectrometers and reference data for various gases is available from the HITRAN database. ${ }^{39,40}$ Using the EC-QCL setup, IR spectra of water vapor have been recorded and the absorption lines were compared to reference data. Figure S3A shows the shift of water vapor bands acquired with two available ADCs relative to the position of vapor bands taken from the reference database. The results indicate that the ADCs introduce a characteristic and non-constant shift to the wavenumber scale. For the AD7760, the deviation is about $+3 \mathrm{~cm}^{-1}$ across the observed wavenumber region, with a small slope $(\mathrm{k}=-0.0083)$, while for the $\mathrm{NI}$ $\mathrm{ADC}$, the aberration is generally smaller $\left(-1 \mathrm{~cm}^{-1}-+3 \mathrm{~cm}^{-}\right.$ ${ }^{1}$ ), but with a larger change across the observed wavenumber region $(\mathrm{k}=0.0408)$. This behavior resulted unchanging for repeated measurements. Due to a lower noise level $\left(\mathrm{RMS}_{\mathrm{AD} 7760}=0.54 \mathrm{mAU}, \mathrm{RMS}_{\mathrm{NI}}=0.37 \mathrm{mAU}\right)$, the $\mathrm{NI}$ $\mathrm{ADC}$ was used for protein measurements. The relation between the recorded and the reference wavenumber scale obtained by the water vapor spectrum was used to calibrate the protein spectra. Figure S-3B shows the efficiency of the wavenumber calibration for a water vapor band. This frequency calibration accounts for all deviations introduced in the experimental setup (EC-QCL, data acquisition). The remaining wavenumber deviation introduced by shifting and warping of the COW processing routine was found to be lower than $0.1 \mathrm{~cm}^{-1}$ which is negligible for liquid phase IR measurements.

Enabling temperature-stabilized measurements. The $v_{2}$-vibration of water ( $\mathrm{HOH}$-bending) with a maximum near $1645 \mathrm{~cm}^{-1}$ overlaps with the amide I band of proteins. ${ }^{41}$ The position of the bending band is rather sensitive to changes of the temperature, as it depends on the strength of the hydrogen bonds. Upon temperature increase, the hydrogen bonds become weaker, leading to a shift of the bending vibration to lower wavenumbers accompanied with narrowing of the band and an increase of intensity. ${ }^{42}$ This behavior results in a rather complex change of the solvent spectrum..$^{43}$ The impact is particularly strong due to the high abundance of water as a solvent and its high molar absorptivity in this region. ${ }^{41}$ Further, it has been reported, that altering temperatures also affect the effective path length of the transmission cell due to the thermal expansion of the spacer material. ${ }^{44}$ Thermal expansion of a $38 \mu \mathrm{m}$ Teflon spacer (linear expansion coefficient $\left.\alpha=0.00012 \mathrm{~K}^{-1}\right)^{45}$ at a temperature change of $1 \mathrm{~K}$ leads to an increase of the path length of $0.005 \mu \mathrm{m}$, resulting in a change of absorbance of $0.57 \mathrm{mAU}$ for the $\mathrm{HOH}$ bending band $\left(\mathrm{c}=55.56 \mathrm{~mol} \mathrm{~L}^{-1}, \varepsilon=22.3086 \mathrm{~L} \mathrm{~mol}^{-1} \mathrm{~cm}^{-1}\right.$ at $1645 \mathrm{~cm}^{-1}$ ). The combination of these effects leads to a change of shape and intensity in the resulting absorbance spectrum, in case the background and sample single beam spectra are recorded at different temperatures. Since for the qualitative assessment of spectral features attributed to protein secondary structure the band shape and relative intensities are important, measurements performed at constant temperatures are essential. To achieve this, a highly constant temperature cell has been designed. The temperature stability of the employed cell lies within $0.005{ }^{\circ} \mathrm{C}$ across a time period of $30 \mathrm{~min}$.

Comparison of mid-IR spectra recorded with the ECQCL setup and a FTIR spectrometer. Figure S-4 shows the $100 \%$ lines of water for the EC-QCL setup and the FTIR instrument at acquisition parameters used for protein measurements. $100 \%$ lines are employed for evaluation of the noise level of a spectrometer setup and are obtained by calculation the absorbance spectrum of two subsequent single beam spectra of the same sample under identical conditions. Under ideal conditions, the result would be a flat line at $100 \%$ transmittance, corresponding to zero absorbance. ${ }^{15}$ The RMS (root mean square) of the $100 \%$ line at path lengths employed for protein measurements is $0.05 \mathrm{mAU}$ for the FTIR setup and $0.37 \mathrm{mAU}$ for the EC-QCL setup. When considering the approx. four times larger absorbance values gained by utilizing a cell with $38 \mu \mathrm{m}$ path length for the EC-QCL setup compared to $8 \mu \mathrm{m}$ in the FTIR spectrometer, the resultant signal to noise ratios (SNR) are in the same order of magnitude (e.g. SNR FTIR $\sim 820$ and $\mathrm{SNR}_{\mathrm{EC}-\mathrm{QCL}} \sim 550$ for $20 \mathrm{mg} \mathrm{mL}^{-1}$ protein solutions). Moreover, for assessing the noise level of a measurement, all contributors need to be considered. Regarding the light source, the noise level introduced by low intensity thermal light sources in FTIR spectrometers can usually be neglected. High intensity laser sources, however, contribute to the overall measurement noise, particularly when operated in pulsed mode. Referring to the detector, the detectivity of the $\mathrm{LN}_{2}$ cooled MCT-detector in the FTIR spectrometer is ten times higher than the peltier-cooled MCT-detector applied in the QCL setup. An important point to consider for a transmission setup is the employed path length. Generally, when sufficient light is passing through the sample and reaching the detector (i.e. low path length) the noise level will essentially remain constant. However, when increasing the path length up until a range where most of the light intensity is absorbed by the sample, the measurement noise will begin to increase disproportionately, ${ }^{2}$ as demonstrated by the $100 \%$ line of water recorded by the FTIR spectrometer utilizing the cell with a path length of $38 \mu \mathrm{m}$ (RMS $=99.94 \mathrm{mAU})$, as demonstrated in Figure S-4. Accordingly, the increase of the path length accompanied with the gain in ruggedness of the analysis system is exclusive to the EC-QCL setup while featuring a comparable SNR to FTIR spectroscopy.

FTIR spectra of the protein solutions were recorded as a reference to demonstrate the equality of the protein IR spectra acquired by EC-QCL with IR spectra recorded by well-established FTIR spectroscopy. Figure 3F-J show the IR spectra of $20 \mathrm{mg} \mathrm{ml}^{-1}$ protein solutions recorded with a path length of $8 \mu \mathrm{m}$. Figure 3 shows that the shape and position of the absorption bands recorded by the EC-QCL setup correspond well with the conventional method. In order to quantify the congruence between the (normalized) spectra, the degree of spectral overlap was computed. Using eq. 2 , the $s_{12}$ values obtained for BSA, Hemo, ACT, BLG and Lys were 0.99569, 0.99668, 0.99959, 0.99853, and 0.99783 , respectively. These figures allow us to conclude that the spectra acquired with the EC-QCL setup contain equivalent information as the FTIR spectra. For further evaluation, second-derivative spectra are shown in Figure 4. This band narrowing technique is commonly 
employed to resolve overlapping bands in complex spectral regions. ${ }^{3,18}$ Second-derivative spectra of protein samples acquired by EC-QCL (Figure 3A-E) show similar spectral features as the ones recorded by FTIR spectroscopy (Figure 3F-J). Also here the mathematical evaluation method to quantify the spectral overlap was applied. $s_{12}$ values of $0.99006,0.97120,0.98534,0.96762$ and 0.98588 for BSA, Hemo, ACT, BLG and Lys, respectively, indicate high spectral overlap, considering the enhanced sensitivity of the second-derivative spectra.

\section{CONCLUSIONS AND OUTLOOK}

A robust setup employing a broadly tunable EC-QCL was introduced for IR transmission measurements of protein solutions. Characteristic spectral features of five proteins with different secondary structures have been successfully measured at concentrations as low as $2.5 \mathrm{mg} \mathrm{mL}^{-1}$. Mathematical evaluation of the spectral overlap and comparison of second derivative spectra confirm excellent match of the QCL transmission measurements with protein spectra acquired by FTIR spectroscopy. The validity of LambertBeer law has been demonstrated for protein concentrations between 1-20 $\mathrm{mg} \mathrm{mL}^{-1}$. To allow for accuracy in the wavenumber scale employing the EC-QCL setup, a calibration method has been elaborated to reference the acquired IR spectra to the absolute positions of the water vapor absorption bands. An advanced data processing routine has been devised to diminish intensity variations inherent to the emission spectra EC-QCLs. In future, arrays of DFBQCLs that provide broadband wavelength tunability without featuring moving parts may represent an important step to overcome the experimental difficulties encountered with EC-QCLs. The high optical power provided by the QCL allowed using significantly higher optical paths $(38 \mu \mathrm{m})$ than applicable with conventional FTIR spectrometers. This increase of accessible optical path length opens a wide range of new experimental possibilities in a spectral region that has been limited due to the high absorbance of water. The larger flow cross section results in a greatly reduced pressure drop in the cell and allows experiments in flow through configuration. That signifies a major step forward for IR investigations of proteins, which mostly have been conducted in $\mathrm{D}_{2} \mathrm{O}$, accompanied with more laborious sample preparation and ambiguities regarding the non-native environment. In future, the introduced setup will be employed to monitor changes of protein secondary structure in aqueous solution induced by heat and chemical denaturation.

\section{ASSOCIATED CONTENT}

\section{Supporting Information}

Figure S-1: Calibration curves for proteins. Figure S-2: Illustration of processing steps for single beam spectra. Figure S-3: Shift of the water vapor absorption bands recorded with the EC-QCL setup compared to the reference positions taken from database. Figure S-4: 100\% lines for EC-QCL setup and FTIR instrument. This material is available free of charge via the Internet at http://pubs.acs.org.

\section{AUTHOR INFORMATION}

\section{Corresponding Author}

*All correspondence should be addressed to Bernhard Lendl (bernhard.lendl@tuwien.ac.at).

\section{Present Addresses}

$\S$ (M.B.) Research Center for Non Destructive Testing GmbH, Altenbergerstraße 69, 4040 Linz, Austria.

\section{Notes}

The authors declare no competing financial interest.

\section{ACKNOWLEDGMENTS}

The authors thank Dieter Baurecht for fruitful discussions. Financial support was provided by the Austrian research funding association (FFG) under the scope of the COMET programme within the research project "Industrial Methods for Process Analytical Chemistry - From Measurement Technologies to Information Systems (imPACts)" (contract \#843546). M.R.A gratefully acknowledges the financial support provided by CONICET.

\section{REFERENCES}

(1) Cooley, J. W.; Tukey, J. W. Math. Comput. 1965, 19, 297301.

(2) Brandstetter, M.; Volgger, L.; Genner, A.; Jungbauer, C.; Lendl, B. Appl. Phys. B: Lasers Opt. 2013, 110, 233-239.

(3) Yang, H.; Yang, S.; Kong, J.; Dong, A.; Yu, S. Nat. Protoc. 2015, 10, 382-396.

(4) Faist, J.; Capasso, F.; Sivco, D. L.; Sirtori, C.; Hutchinson, A. L.; Cho, A. Y. Science 1994, 264, 553-556.

(5) Weida, M. J.; Yee, B. In Imaging, Manipulation, and Analysis of Biomolecules, Cells, and Tissues IX; Farkas, D. L.; Nicolau, D. V.; Leif, R. C., Eds; Proc. SPIE: San Francisco, 2011; pp $1-7$.

(6) Yao, Y.; Hoffman, A. J.; Gmachl, C. F. Nat. Photonics 2012, 6, 432-439.

(7) Capasso, F. Opt. Eng. 2010, 49.

(8) Curl, R. F.; Capasso, F.; Gmachl, C.; Kosterev, A. A.; McManus, B.; Lewicki, R.; Pusharsky, M.; Wysocki, G.; Tittel, F. K. Chem. Phys. Lett. 2010, 487, 1-18.

(9) Weidmann, D.; Tittel, F. K.; Aellen, T.; Beck, M.; Hofstetter, D.; Faist, J.; Blaser, S. Appl. Phys. B: Lasers Opt. 2004, 79, 907-913.

(10) Shi, Q.; Nelson, D. D.; McManus, J. B.; Zahniser, M. S.; Parrish, M. E.; Baren, R. E.; Shafer, K. H.; Harward, C. N. Anal. Chem. 2003, 75, 5180-5190.

(11) Nagele, M.; Hofstetter, D.; Faist, J.; Sigrist, M. W. Anal. Sci. 2001, 17, S497-S499.

(12) Wysocki, G.; McCurdy, M.; So, S.; Weidmann, D.; Roller, C.; Curl, R. F.; Tittel, F. K. Appl. Opt. 2004, 43, 6040-6046.

(13) Siegmann-Hegerfeld, T.; Genner, A.; Brandstetter, M.; Miltner, M.; Lendl, B.; Harasek, M. Chem. Eng. Trans. 2013, 35, 979-984.

(14) Brandstetter, M.; Genner, A.; Anic, K.; Lendl, B. Analyst 2010, 135, 3260-3265.

(15) Brandstetter, M.; Lendl, B. Sensors Actuators B Chem. 2012, 170, 189-195.

(16) Brandstetter, M.; Sumalowitsch, T.; Genner, A.; Posch, A. E.; Herwig, C.; Drolz, A.; Fuhrmann, V.; Perkmann, T.; Lendl, B. Analyst 2013, 138, 4022-4028.

(17) Barth, A. Biochim. Biophys. Acta 2007, 1767, 10731101.

(18) Fabian, H.; Mäntele, W. In Handbook of Vibrational Spectroscopy; Chalmers, J. M.; Griffiths, P. R., Eds.; John Wiley \& Sons, Ltd: Chichester, 2002; pp 3399-3425.

(19) Bal Ram, S. In Infrared Analysis of Peptides and Proteins; Bal Ram, S, Ed; American Chemical Society: Washington, 1999; pp 2-37. 
(20) Goormaghtigh, E.; Ruysschaert, J. M.; Raussens, V. Biophys. J. 2006, 90, 2946-2957.

(21) Jackson, M.; Mantsch, H. H. Crit. Rev. Biochem. Mol. Biol. 1995, 30, 95-120.

(22) Oberg, K. A.; Ruysschaert, J. M.; Goormaghtigh, E. Eur. J. Biochem. 2004, 271, 2937-2948.

(23) Ramer, G.; Balbekova, A.; Schwaighofer, A.; Lendl, B. Anal. Chem. 2015, 87, 4415-4420.

(24) Kong, J.; Yu, S. Acta Biochim. Biophys. Sin. 2007, 39, 549559.

374.

(25) Bouhekka, A.; Bürgi, T. Appl. Surf. Sci. 2012, 261, 369-

(26) Das, D. K.; Mondal, T.; Mandal, U.; Bhattacharyya, K. ChempPhysChem 2011, 12, 814-822.

(27) Rial, E.; Muga, A.; Valpuesta, J. M.; Arrondo, J.-L. R.; Goñi, F. M. Eur. J. Biochem. 1990, 188, 83-89.

(28) Arrondo, J. L. R.; Goñi, F. M. Prog. Biophys. Mol. Biol. 1999, 72, 367-405.

(29) Wagner, C.; Genner, A.; Ramer, G.; Lendl, B. In Modeling, Programming and Simulations Using LabVIEW; De Asmundis, R., Ed; InTech: Rijeka, 2011; pp 1-20.

(30) Jellema, R. H. In Comprehensive Chemometrics: Chemical and Biochemical Data Analysis; Brown, S.D; Tauler, R. Walczak, B., Eds; Elsevier: Oxford, 2010; pp 85-108.

(31) Culzoni, M. J.; Goicoechea, H. C.; Ibañez, G. a.; Lozano, V. a.; Marsili, N. R.; Olivieri, A. C.; Pagani, A. P. Anal. Chim. Acta 2008, 614, 46-57.

(32) Levitt, M.; Greer, J. J. Mol. Biol. 1977, 114, 181-239.

(33) Grdadolink, J.; Marechal, Y. Biopolymers 2001, 62, 4053.

(34) Monaco, H. L.; Zanotti, G.; Spadon, P.; Bolognesi, M.; Sawyer, L.; Eliopoulos, E. E. J. Mol. Biol. 1987, 197, 695-706.

(35) Goormaghtigh, E.; Cabiaux, V.; Ruysschaert, J. M. Eur. J. Biochem. 1990, 193, 409-420.

(36) Wysocki, G.; Curl, R. F.; Tittel, F. K.; Maulini, R.; Bulliard, J. M.; Faist, J. Appl. Phys. B: Lasers Opt. 2005, 81, 769-777.

(37) Brandstetter, M.; Koch, C.; Genner, A.; Lendl, B. In Quantum Sensing Nanophotonic Devices Xi. Razeghi, M.; Tournié, E.; Brown, G. J., Eds; Proc. SPIE: San Francisco, 2014, pp 1-11.

(38) Echabe, I.; Encinar, J. A.; Arrondo, J. L. R. Biospectroscopy 1997, 3, 469-475.

(39) Hanssen, L. M.; Zhu, C. In Handbook of Vibrational Spectroscopy; Chalmers, J. M., Griffiths, P. R., Eds.; John Wiley \& Sons Ltd: Chichester, 2002; pp 881-890.

(40) Rothman, L. S.; Gordon, I. E.; Babikov, Y.; Barbe, A.; Benner, D. C.; Bernath, P. F.; Birk, M.; Bizzocchi, L.; Boudon, V.; Brown, L. R.; Campargue, A.; Chance, K.; Cohen, E. A.; Coudert, L. H.; Devi, V. M.; Drouin, B. J.; Fayt, A.; Flaud, J. M.; Gamache, R. R.; Harrison, J. J.; Hartmann, J. M.; Hill, C.; Hodges, J. T.; Jacquemart, D.; Jolly, A.; Lamouroux, J.; Le Roy, R. J.; Li, G.; Long, D. A.; Lyulin, O. M.; Mackie, C. J.; Massie, S. T.; Mikhailenko, S.; Muller, H. S. P.; Naumenko, O. V; Nikitin, A. V; Orphal, J.; Perevalov, V.; Perrin, A.; Polovtseva, E. R.; Richard, C.; Smith, M. A. H.; Starikova, E.; Sung, K.; Tashkun, S.; Tennyson, J.; Toon, G. C.; Tyuterev, V. G.; Wagner, G. J. Quant. Spectrosc. Radiat. Transfer 2013, 130, 4-50.

(41) Venyaminov, S. Y.; Prendergast, F. G. Anal. Biochem. 1997, 248, 234-245.

(42) Praprotnik, M.; Janežič, D.; Mavri, J. J. Phys. Chem. A 2004, 108, 11056-11062.

(43) Freda, M.; Piluso, A.; Santucci, A.; Sassi, P. Appl. Spectrosc. 2005, 59, 1155-1159.

(44) Amunson, K. E.; Anderson, B. A.; Kubelka, J. Appl. Spectrosc. 2011, 65, 1307-1313.

(45) Smith, R.; Ellis, B. Polymers, 2nd ed.; CRC Press: Boca Raton, 2008; pp 333-1027. 


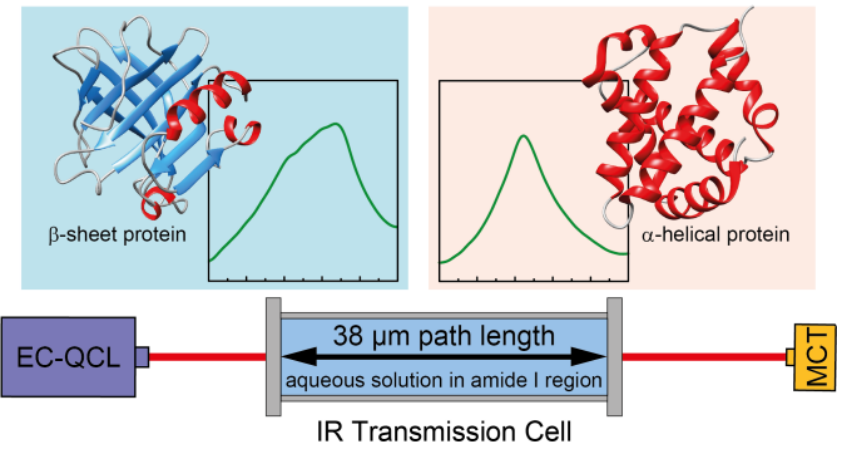

